\section{Visual fields in patients with multifocal intraocular lens implants and monovision: an exploratory study}

\begin{abstract}
Purpose To assess the difference in binocular visual fields (VFs) in patients who underwent bilateral cataract surgery with either multifocal (MF; Tecnis ZM900, AMO) intraocular lenses (IOLs) or monofocal IOLs with powers adjusted to give monovision (MV; Akreos AO, Bausch\&Lomb). Setting St George's \& Moorfields Eye Hospital, London.

Methods Prospective exploratory study. Binocular Esterman VFs (Humphrey Field Analyser II) were compared between $\mathbf{1 0}$ participants with MV and 16 participants with MF IOLs. The dominant eye in MV participants had 0 to $-0.50 \mathrm{DS}$ and the nondominant eye had between -1.0DS and -1 .5DS. Best-corrected Snellen visual acuity for all 52 eyes was six out of nine or better. The main outcome measure was Esterman Efficiency Score. Incidence of suboptimal VF results ( $\geqslant 1$ Unseen Locations - ULs) and mean testing times in the two groups were compared. Results There was no statistically significant difference in the incidence of suboptimal VF results in these two groups $(P=0.662)$.

Test durations in the two groups were not significantly different $(P=0.650)$. However, $3 / 10 \mathrm{MV}$ plots $(33 \%)$ had markedly suboptimal right hemi-fields (distance-dominant eye) compared with 0/15 MF plots. Additionally, the MV group accounted for $79 \%$ of total ULs (20/29) and all these ULs were distributed in areas outside or bordering the true binocular VFs.

Conclusions All included VFs met the UK driving standards criteria. The pattern of VF defects encountered in the MV group is of
\end{abstract}

N Stanojcic ${ }^{1,2}$, M Wilkins $^{2}$, C Bunce ${ }^{2}$ and A lonides $^{2}$

interest because the majority of ULs (13/20; $65 \%$ ) corresponded to the monocular VFs of the distance-dominant eye.

Eye (2010) 24, 1645-1651; doi:10.1038/eye.2010.104; published online 20 August 2010

Keywords: visual fields; Esterman; cataract surgery; multifocal; monovision

\section{Introduction}

The development of multifocal (MF) intraocular lens (IOL) implants has allowed suitable cataract patients to enjoy many benefits that conventional IOLs lack. This means that patients may become independent of any form of external optical appliance following cataract surgery. Patients are expected to have satisfactory vision at distance and near with a reduced need for glasses. This may offer increased independence for activities of daily living and an improvement in the quality of life for suitable candidates.

Monovision (MV) is a technique originally used in contact lens practise to provide presbyopic correction for suitable patients. It works on a principle of simultaneous vision where one eye is corrected for distance and the other for near vision, in the hope that binocular vision will be reasonably good at all distances. This technique has been used in cataract surgery for pseudophakic presbyopes. ${ }^{1-3}$

Patients implanted with MF IOLs commonly report glare and halos that cause them problems during driving at night. ${ }^{4-6}$ In one prospective randomized trial MF IOL patients required on average about $0.20 \log$ units $(2 \mathrm{~dB})$ or about $30 \%$
${ }^{1}$ St George's University of London, Cranmer Terrace, London, UK

${ }^{2}$ Moorfields Eye Hospital NHS Foundation Trust, London, UK

Correspondence:

N Stanojcic, Duke Elder Eye Clinic, St George's Hospital, Lanesborough Wing, Blackshaw Road, London SW17 OQT, UK Tel: + 44 (0)208 785 4556; Fax: + $44(0) 2087854556$ E-mail: nstanojcic@ doctors.org.uk

Received: 16 February 2010 Accepted in revised form: 15 June 2010 Published online: 20 August 2010

This work was presented at the XXVII Congress of the ESCRS Barcelona, Spain, September 2009 
more contrast to achieve the same acuity level as monofocal IOL patients. ${ }^{6}$

Research has shown that there are links between visual problems and crash involvement, self-reported driving difficulties, poor driving simulator performance, and poor on-road driving. ${ }^{7}$ Drivers with restricted fields may be prone to a higher incidence of side collisions. ${ }^{8}$

Previous studies have investigated a number of outcome measures in cohorts of MF and MV patients. These have included best-corrected and uncorrected near and distance visual acuities, depth of field, contrast sensitivity, objective glare, self-reported satisfaction with vision, and so on. ${ }^{2,5,6,9}$ One study that has compared monocular visual fields (VFs) of MF IOL patients (AcrySof ReSTOR, Alcon, Fort Worth, TX, USA) with monofocal IOLs found that mean threshold was lower in the MF group, but this was not statistically significant. ${ }^{10}$

We have found no studies investigating binocular or functional VFs in MF and MV pseudophakic patients.

The purpose of this study was to assess the difference in binocular VFs in patients who have undergone bilateral cataract surgery with either MF IOLs (Tecnis ZM900, AMO, Santa Ana, CA, USA) or with monofocal IOLs with the powers adjusted to give MV (Akreos AO, Bausch\&Lomb, Rochester, NY, USA).

\section{Materials and methods}

\section{Study design}

This was a prospective exploratory study comprising two groups of patients who had undergone bilateral sequential cataract surgery with either MF (Tecnis ZM900, AMO) IOLs or with monofocal IOLs with the powers adjusted to give MV (Akreos AO, Bausch\&Lomb).

\section{Participants}

Participants were identified from an ongoing randomized controlled clinical trial, ${ }^{1}$ where patients had undergone bilateral cataract surgery with either MF IOL or monofocal IOLs with the powers adjusted to give MV. Our study was approved by the Moorfields \& Whittington Research Ethics Committee (UK). Informed written consent was obtained from all participants.

No participant had significant eye co-pathology. All participants took part in our study at least 6 months after their second cataract operation and none experienced or were aware of any post-operative complications or unexpected visual symptoms at the time of VF testing. All participants reported moderate to high satisfaction with their vision and none enquired about or requested IOL explantation. There was no evidence of any posterior capsular opacity (PCO) in either eye at the time of their routine follow-up following the second surgery (3-4 months afterwards). Age of participants in the MF group was $66.4 \pm 12.6$ (mean $\pm \mathrm{SD}$; range $42-88$; median 64) and in the MV group was $68.8 \pm 10.5$ (range 54-82; median 66.5). The MF group comprised 10 female and 6 male participants. The MV group comprised six female and four male participants.

\section{External optical aids}

No patients in either group wore glasses or contact lenses during the binocular VF testing.

\section{Best-corrected/unaided visual acuities/refraction}

Best-corrected Snellen visual acuity in each eye was six out of nine or better for all participants (post-operatively in both groups). Patients in the MF group achieved six out of nine or better with each eye unaided. The spherical error for pre-operatively hyperopic patients was 0 to $+0.5 \mathrm{DS}$ and for pre-operatively myopic patients 0 to $-0.5 \mathrm{DS}$ per eye in the MF group. The cylindrical error was no more than $\pm 1 \mathrm{DC}$ per eye post-operatively in the same group. In the MV group, unaided distance vision of the non-dominant (or near-dominant) eye was between 6/12 and 6/18 (best-corrected Snellen visual acuity 6/9). In the near-dominant eyes, the refraction ranged between $-1.00 \mathrm{DS}$ and $-1.50 \mathrm{DS}$ with no more than 1DC. The dominant eye (or distance-dominant) had 0 to $-0.50 \mathrm{DS}$ with no more than 1DC.

\section{Setting}

VF testing was performed at two locations: St George's Hospital, London and Moorfields Eye Hospital, London. Tests were done in dedicated VF testing rooms at both locations.

\section{VF testing}

VF testing was performed with Humphrey Field Analyzer II (HFA; Humphrey Systems, Dublin, CA, USA), models 745 and 740. All assessments were carried out by the same examiner (the first author). Each participant spent several minutes in the testing room before starting the test. A standardized set of instructions was given to all participants before the test to read and their understanding of the instructions was checked. A brief informal conversation regarding the patients' overall satisfaction with their IOLs normally took place beforehand. Room lighting was dimmed during the test. For each patient a short demonstration test was performed regardless of whether the patient had done the test before or not. 
Fixation monitoring in this study was carried out visually by the examiner (as the blind spot could not be tested automatically), and false positive and false negative results were provided by the HFA. The main outcome measure was Esterman Efficiency Score. The incidence of suboptimal VF results (VF plots with one or more unseen locations-ULs) in the two groups was calculated and compared. In addition, the distributions of ULs in the hemi-fields (superior, inferior, right, and left) were analysed and compared. We also compared mean testing times in the two groups.

\section{Statistical analysis}

As this was an exploratory study, no previous hypothesis was chosen and no sample size was calculated. The incidence of suboptimal binocular Esterman VF result $(\geqslant 1 \mathrm{ULs})$ in the two groups was compared by using the Fisher's Exact test (5\% level). Test durations in the two groups were compared using the Student's $t$-test (5\% level).

We certify that all applicable institutional and governmental regulations concerning the ethical use of human volunteers were followed during this research.

\section{Results}

In all, 25/26 participants (96\%) demonstrated optimal central VFs ( $30^{\circ}$ from fixation). There was one grossly abnormal result. The Esterman Efficiency Score for this patient was 80 (ie, $23 \mathrm{ULs}$ ) and the test time $6 \mathrm{~min} 4 \mathrm{~s}$ (6:04; cf. MF group mean \pm SD: 4:05 \pm 11 ). This participant demonstrated significantly impaired psychomotor ability at the time of the test. It later transpired that he was on high-dose multidrug therapy for his psychiatric condition. It was therefore decided that this result be excluded from the main results analysis. Only 2 of the total 29 ULs $(6.9 \%)$ were below the horizontal midline (from all the $25 \mathrm{VF}$ plots considered). Test durations in the MF and MV groups were 4 min $5 \mathrm{~s} \pm 11 \mathrm{~s}$ (mean $\pm \mathrm{SD}$; range 3:52-4:29) and $4 \mathrm{~min} 2 \mathrm{~s} \pm 15 \mathrm{~s}$ (range 3:37-4:26), respectively. No patients required breaks during the test. There was no statistically significant difference in testing times between these two groups $(P=0.650)$.

A total of 15 plots were considered and analysed in the MF group. All patients had optimal fixation on visual monitoring. False positive errors occurred in only two plots and were within the accepted limit. ${ }^{11}$ Minimal false negative errors occurred only in one plot. There were six suboptimal VF plots in total $(6 / 15 ; 40 \%)$. These accounted for a total of nine ULs. All the ULs were in the area outside or bordering the true binocular VFs (ie, the theoretical monocular VFs, shown as grey areas in Figure 1). Four plots demonstrated a single UL in approximately the same VF location (red fields in Figure 2). One plot had a single UL in the left superior quadrant (green field in Figure 2). Another plot accounted for the remaining four ULs (yellow fields in Figure 2).

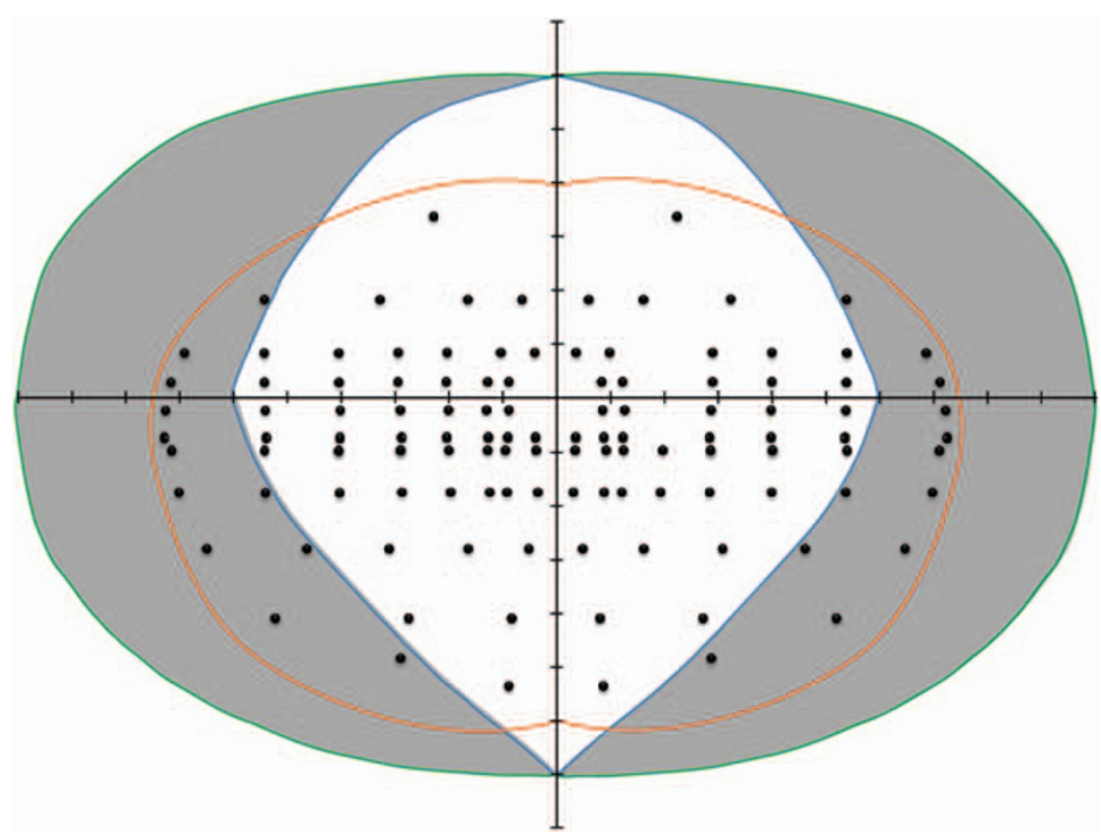

Figure 1 Binocular visual fields in relation to a typical HFA II Esterman field plot. Green line delineates the normal extent of binocular visual field. Blue line delineates approximation of the true binocular visual field. Orange line delineates the area tested by the Esterman binocular visual field test. Grey area represents approximation of the purely monocular visual fields. The graduations on the ordinate and abscissa represent $10^{\circ}$ steps. 


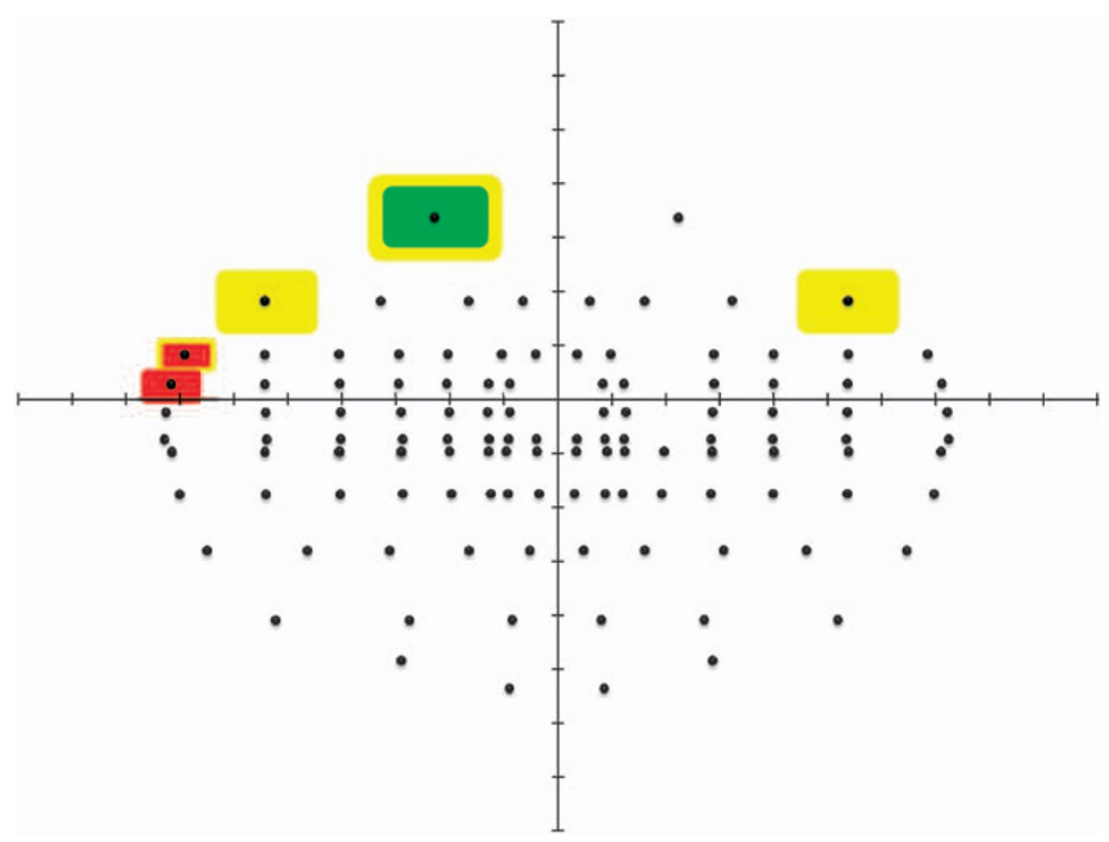

Figure 2 Areas of visual field loss in the MF group. Four participants had only one UL each (one of the two red fields), accounting for four ULs. One plot accounted for four ULs (yellow fields). One plot demonstrated only one UL (green field).

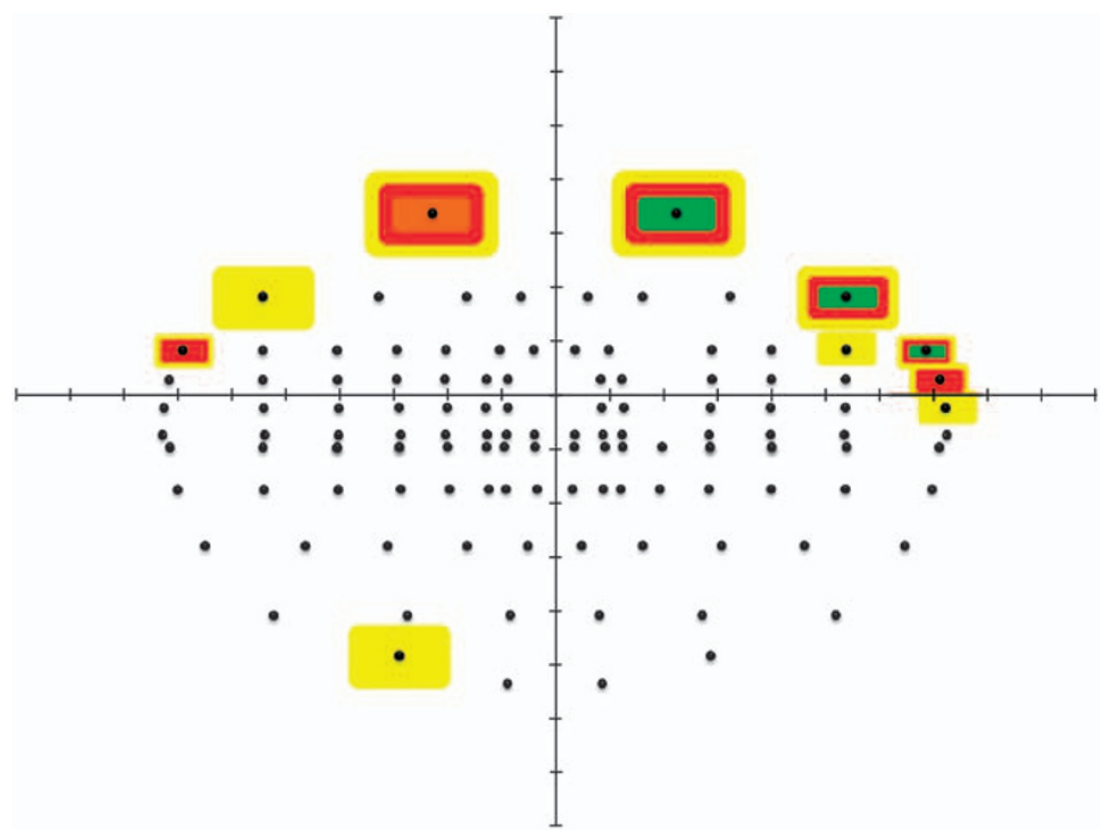

Figure 3 Areas of visual field loss in the monovision group. Three patients were right-eye dominant and had VF loss predominantly in the right upper VFs (areas coloured yellow, red, and green). Area coloured amber corresponds to the single UL of one patient.

A total of 10 plots were analysed in the MV group. There was a total of 20 ULs from four suboptimal plots $(4 / 10 ; 40 \%)$. Both visually monitored fixation and automatically recorded reliability indices (ie, false positive and false negative errors) were optimal in this group. All the ULs were confined to areas outside or bordering the true binocular VFs (grey areas in Figure 1). A total of 13 ULs $(13 / 20 ; 65 \%)$ corresponded to the monocular VFs of the distance-dominant eye. For three candidates, ULs were predominantly or exclusively distributed in their distance-dominant-eye VFs (ie, the right superior quadrant in Figure 3). One participant had 
a single UL in the left superior quadrant (shown amber in Figure 3). One plot accounted for 10 ULs (shown yellow in Figure 3). Another plot accounted for six ULs (shown red in Figure 3). One plot had three ULs (shown green in Figure 3)

There was no statistically significant difference in the incidence of suboptimal binocular VF results (ie, VF plots with one or more ULs) in these two groups that had received different treatments $(P=0.662)$.

\section{Discussion}

In this study, we examined binocular VFs of two groups of patients who had undergone bilateral sequential cataract surgery with either MF (Tecnis ZM900) IOLs or monofocal IOLs with powers adjusted to give MV (Akreos AO). All included VF plots $(25 / 26)$ met the UK driving standards criteria. ${ }^{11}$ No patients in this study had facial characteristics that could have significantly restricted their VFs (eg, deep set eyes, prominent brows). No ptosis encroaching the pupil was observed in any of the participants. Careful consideration was given to positioning the patients at the HFAII to avoid artefacts caused by bad posture. Each participant's head was carefully positioned on the chin-rest to optimize their VFs. Pupil diameters were not formally measured, and this is a limitation of our study. Miosis is therefore certainly a factor that may have affected the VFs, especially in the older patients.

Esterman Binocular protocol was used to assess the participants' functional VFs, as binocular VF testing is thought to be the best indicator of a patient's functional VFs. ${ }^{12,13}$ The Driver and Vehicle Licensing Agency in the UK use results of this test to decide patients' eligibility to hold a driving licence. ${ }^{11}$ The Esterman is a relatively quick wide-field test. ${ }^{14}$ This was considered particularly important as prolonged testing times are known to affect subject performance. Excessive fatigue or malaise caused either by the patient's underlying physical state or by an overly demanding VF examination, can render results of VF examination useless. The simplest and most direct method of gaining the necessary amount of information should therefore be employed whenever possible. ${ }^{15}$

Test durations in our study were $4 \mathrm{~min} 5 \mathrm{~s} \pm 11 \mathrm{~s}$ (mean \pm SD) in the MF group and $4 \mathrm{~min} 2 \mathrm{~s} \pm 15 \mathrm{~s}$ in the MV group. Our patients were therefore expected to be able to maintain adequate concentration levels during the testing, resulting in reliable VF plots.

Esterman protocol uses a Goldmann target III4e. Size III Goldman target is thought to be small enough to find even very small scotomas and yet large enough to be relatively unaffected by residual refractive error. ${ }^{16}$ During the Esterman VF test, each of the 120 tested points is tested twice and is only marked as an unseen point where the patient fails to respond to the stimuli on both occasions. ${ }^{8}$ The Esterman binocular programme assesses 120 points and extends $75^{\circ}$ nasally, $75^{\circ}$ temporally, $40^{\circ}$ superiorly, and $60^{\circ}$ inferiorly. In theory, this covers a large area of normal human VFs. For a bright stimulus, the usual extent of binocular VFs is $60^{\circ}$ superiorly, $70-75^{\circ}$ inferiorly, $100^{\circ}$ temporally, and $60^{\circ}$ nasally. ${ }^{16,17}$ The approximate extent of normal binocular VFs is shown by the green line in Figure 1.

Almost all the ULs (27/29; 93\%) observed in our study were in the superior hemi-field. In addition, all the 27 ULs were outside the central $30^{\circ}$. Abnormal VFs were generally most restricted superiorly (beyond $\sim 30^{\circ}$ from fixation) and slightly less restricted horizontally (beyond $\sim 45^{\circ}$ from fixation).

It was not expected that our two groups of patients without significant eye co-pathology, would demonstrate ULs in the area of the true binocular VFs (Figure 1). This was however the case with the superior VFs, where ULs were observed even in the true binocular VFs. Apart from the possible effect of miosis, there may be other explanations for this VF loss.

A relatively large cross-sectional study (562 normal eyes) found that influence of age on VF sensitivity was both eccentricity and hemi-field dependant. ${ }^{18}$ Small but significantly larger rates of sensitivity loss were identified both peripherally and superiorly. However, although age has a significant effect on mean VF sensitivity, about $75 \%$ of the sensitivity variation still remains unaccounted for. ${ }^{18}$

The MV group in our study accounted for $79 \%$ of the total ULs (ie 20/29). All of the 20 ULs in the MV group were distributed in areas outside or bordering the true binocular VFs. In addition, the pattern of this binocular VF loss showed some interesting characteristics.

In all, 3/10 VF plots (33\%) in the MV group had markedly suboptimal right hemi-fields (corresponding to the distance-dominant eye) compared with $0 / 15 \mathrm{VF}$ plots in the MF group (Figures 2 and 3). All participants in the MV group were right-eye dominant (thus the same eye was emmetropic or slightly myopic). There was only one participant in the MF group (1/16), whose plot suggested a suboptimal left hemi-field loss (three ULs in the left hemi-field showed in yellow in Figure 2).

As mentioned above, patients in the MV group had between $-1.0 \mathrm{DS}$ and 1.5DS in their near-dominant eye and 0 to -0.50 in the distance-dominant eye (with no more than 1DC in each eye). At the testing distance (ie $33 \mathrm{~cm}$ ), the optimum refraction would be $-3.0 \mathrm{DS}$ in each eye. The three patients in the MV group who had ULs in the right superior hemi-field (corresponding to their distance-dominant eye) were 58 years old and older (namely 58, 63, and 74). It is therefore unlikely that any of these patients had significant useful amplitudes of 
accommodation. These three patients were therefore likely to experience blur of between -2.00 and $-3.0 \mathrm{D}$ spherical equivalent at the testing distance in their distance-dominant eye. It is also likely that these patients' near-dominant eye was also relatively blurred (by between -1.0 and 2.0D spherical equivalent) at the testing distance. This may help explain several ULs corresponding to their near-dominant-eye VFs (Figure 2). However, some participants in the MV group had a spherical equivalent approaching $-1.0 \mathrm{D}$ in their distance-dominant eye rendering them myopic to a similar degree in both eyes, thus minimizing the benefit of MV.

Koller et $a l^{19}$ suggested that in the peripheral VFs (outside the central $30^{\circ}$ ), refractive errors have a minor influence on the decrease of the differential light sensitivity because of significant spatial summation through the relatively greater contribution of rods. However, this 'minor influence' was not quantified, and the study in question ${ }^{19}$ only examined the peripheral VFs between 30 and $50^{\circ}$ from fixation. Furthermore, there appears to be no accurate data in the literature about the degree by which differential light sensitivity is affected by refractive errors beyond $50-60^{\circ}$ from fixation.

The distribution of ULs in the MV group did not demonstrate uniform VF constriction. The ULs were mainly in the superior VFs of the distance-dominant eye. It is possible that a combination of aging factors and relative refractive blur both contributed to the VF plot patterns observed in this group. Psychological factors may have also had a part despite the measures taken to optimize the participants' performance during testing.

Patients with MF IOLs on the other hand were not expected to show any near or distance-dominant-eye blur because of the optical properties of MF IOLs (a diffractive aspheric design in this study).

Variation in normal peripheral VFs has previously been investigated with Goldmann kinetic perimeter (22 subjects aged 19-42). ${ }^{20}$ The subjects had refractions between -8.0DS and +8.0DS and normal ophthalmological examination. Testing was performed without corrective glasses and one eye was randomly chosen from each subject. Results for four Goldmann targets (V4e, III4e, I4e, and I3e) were then plotted.

According to Niederhausen and Mojon ${ }^{20}$, normal extent of the superior temporal VF quadrant may lie anywhere between 20 and $70^{\circ}$ from fixation. In view of these findings, quite a few of the ULs observed in our study may be explained as normal variation. However, the data from Niederhausen and Mojon ${ }^{20}$ suggest that the temporal field extends beyond $90^{\circ}$ along and between the 15 and $345^{\circ}$ meridians, for a III4e target. Number of missed points in our study were located between the
15 and $345^{\circ}$ meridians, and the corresponding Goldmann plot $^{20}$ does not offer an explanation for this.

A literature review of MV contact lens studies reported that static VFs were not adversely affected in MV contact lens wearers. ${ }^{21}$ It was found that the peripheral VF width was slightly better (by $1-3^{\circ}$ ) in the eye corrected for near (the non-dominant eye) compared with the eye corrected for distance (the dominant eye). This was thought to be normal variation in measurements. ${ }^{21}$ The levels of VF changes reported in this literature review are therefore too small to account for the levels observed in our study.

However, effects of the surgical procedure itself may help provide an explanation. In a survey by American Society of Cataract and Refractive Surgery and European Society of Cataract and Refractive Surgeons, decentration was found to be the top cause for explantation of hydrophobic acrylic, silicon, and collamer lenses of one piece and three piece design (Ophthalmology Times, published on 4 April 2009). The same report stated that opacification and calcification were the leading causes for explanation of hydrophilic or hydrogel lenses. As the monofocal IOL used in the MV group of our study was an acrylic and hydrophilic IOL (Akreos AO), this may be one explanation for the VF results we observed. PCO, the incidence of which is about $10 \%$ at 2 years post-operatively, ${ }^{22}$ could be another explanation for the suboptimal VF plots that we encountered.

As impaired visual acuity is the main concern in PCO, studies of PCO normally report presence of PCO in the central few millimetres of the posterior capsular pole. It is therefore likely that the incidence of PCO in the periphery of the posterior capsule is more common. Although it is unlikely to affect the visual axis, PCO is likely to affect the peripheral VFs much earlier than the visual axis.

Tilt and decentration of IOLs are known to deteriorate image quality. Decentration and tilt induce higher order aberrations and particularly third order aberrations (coma). ${ }^{23}$ Although the aspheric IOLs have an advantage of reducing undesirable aberrations, these advantages are cancelled if the IOL is decentred. ${ }^{23} \mathrm{MF}$ IOL implanted in the MF group in our study may have been decentred in some subjects. It is also possible that decentration may have an effect on differential light sensitivity in the periphery and therefore on the pattern of VF results observed in both the MF and MV groups.

\section{Conclusion}

This was an exploratory study involving 26 patients who had undergone bilateral sequential cataract surgery with either MF IOLs or monofocal IOLs, with powers adjusted to give MV. There was no statistically significant difference between our two groups in terms of incidence 
of suboptimal binocular VF plots (ie VF plots with one or more ULs). However, the pattern of suboptimal VF results encountered in the MV group is of interest because the majority of ULs corresponded to the monocular VFs of the distance-dominant eye.

A larger study is needed to investigate peripheral VF sensitivity in patients with bilateral MF IOLs and monofocal IOLs with powers adjusted to give MV. This may be done in the same way as in our study with an Esterman Binocular test or using a similar, possibly a full threshold, wide-field peripheral VF test. It may be sufficient to only test the VFs beyond $30^{\circ}$ from fixation. Further studies may also benefit from a control group of 'standard' monofocal patients (with IOL powers adjusted for distance vision) with appropriate correction for the VF testing distance.

\section{Summary}

\section{What was known before}

- We are not aware of any studies that have previously examined binocular or functional visual fields (VFs) in patients who underwent bilateral cataract surgery with either multifocal intraocular lenses (IOLs) or monofocal IOLs with powers adjusted to give monovision (MV).

\section{What this study adds}

- All VF plots we included in our study met the UK driving standards criteria. The suboptimal VF plots amongst these, invariably demonstrated unseen locations (ULs) only outside central $30^{\circ}$ (from fixation).

- The characteristics of suboptimal VF results encountered in our MV group are of interest because the majority of ULs corresponded to the monocular VF of the distancedominant eye.

- The results of our study may help plan and power future studies in the same field.

\section{Conflict of interest}

The authors declare no conflict of interest.

\section{Acknowledgements}

We would like to thank the staff at Moorfields St George's Hospital and the Glaucoma Research Unit at Moorfields City Road which kindly helped with testing arrangements. We would also like to thank the people who volunteered to take part in this study. The Study was sponsored by Moorfields Eye Hospital London, NHS Trust, England, UK.

\section{References}

1 Wilkins M, Allan B, Rubin G. A prospective randomized controlled trial comparing monovision with a multifocal implant in patients undergoing bilateral cataract surgery. 2006, Available at: http://www.ioltrial.org.
2 Leyland M, Zinicola E. Multifocal versus monofocal intraocular lenses in cataract surgery: a systematic review. Ophthalmology 2003; 110: 1789-1798.

3 Greenbaum S. Monovision pseudophakia. J Cataract Refract Surg 2002; 28: 1439-1443.

4 Talley-Rostov A. Patient-centred care and refractive cataract surgery. Curr Opin Ophthalmol 2008; 19: 5-9.

5 Mester U, Hunold W, Wesendahl T, Kaymak H. Functional outcomes after implantation of Tecnis ZM900 and Array SA40 multifocal intraocular lenses. J Cataract Refract Surg 2007; 33: 1033-1040.

6 Steinert RF, Post CT, Brint SF, Fritch CD, Hall DL, Wilder LW et al A prospective, randomized, double-masked comparison of a zonal-progressive multifocal intraocular lens and a monofocal intraocular lens. Ophthalmology 1992; 99: 853-861.

7 Babizhayev MA. Glare disability and driving safety. Ophthalmic Res 2003; 35: 19-25.

8 Rowe F. Visual Fields via the Visual Pathway. Blackwell Publishing Ltd: Oxford, England, 2006; 60-71.

9 Nijkamp MD, Dolders MGT, de Brabander J, van der Borne B, Hendrikse F, Nuijts RMMA. Effectiveness of multifocal intraocular lenses to correct presbyopia after cataract surgery. Ophthalmology 2004; 111: 1832-1839.

10 Bi H, Cui Y, Ma X, Cai W, Wang G, Ji P et al Early clinical evaluation of AcrySof ReSTOR multifocal intraocular lens for treatment of cataract. Ophthalmologica 2008; 222: 11-16.

11 DVLA: at a glance guide to the current medical standards of fitness to drive. September 2009, Available at: http://www.dft.gov.uk/dvla/ /media/pdf/medical/ at_a_glance.ashx.

12 Esterman B. Functional Scoring of the binocular field. Ophthalmology 1982; 89: 1226-1234.

13 Mills RP, Drance SM. Esterman disability rating in severe glaucoma. Ophthalmology 1986; 93(3): 371-378.

14 Jampel HD, Friedman DS, Quigley H. Correlation of the binocular visual field with patient assessment of vision. Invest Ophthalmol Vis Sci 2002; 43: 1059-1067.

15 Harrington DO, Drake MV. The visual Fields: Text and Atlas of Clinical Perimetry, 6th edition. Mosby Company: St Louis, Missouri, USA, 1990; 102-103.

16 Haley MJ (ed). The Field Analyser Primer, 2nd edition. Humphrey Systems: Dublin, California, USA, 1993; 6-11.

17 Henson DB. Visual Fields, 2nd edition. Reed Educational and Professional Publishing Ltd: Oxford, UK, 2000; 2-8: 120-121-150-151.

18 Spry PGD, Johnson CA. Senescent changes of the normal visual field: an age-old problem. Optom Vis Sci 2001; 78(6): 436-441.

19 Koller G, Haas A, Zulauf M, Koerner F, Mojon D. Influence of refractive correction on peripheral visual field in static perimetry. Graefes Arch Clin Exp Ophthalmol 2001; 239: 759-762.

20 Niederhausen S, Mojon DS. Normal isopter position in the peripheral visual field in goldmann kinetic perimetry. Ophthalmologica 2002; 216: 406-408.

21 Jain S, Ou R, Azar D. Monovision outcomes in presbyopic individuals after refractive surgery. Ophthalmology 2001; 108: 1430-1433.

22 Denniston AKO, Murray PI. Oxford Handbook of Ophthalmology, 1st edition, Oxford University Press: UK, 2006; 261.

23 Mester U, Sauer T, Kaymak H. Decentration and tilt of a single-piece aspheric intraocular lens compared with the lens position in young phakic eyes. March 2009. J Cataract Refract Surg 2009; 35(3): 485-490. 